\title{
GLAD!
}

Revue sur le langage, le genre, les sexualités

$04 \mid 2018$

Rhétoriques antiféministes

\section{Sara Garbagnoli \& Massimo Prearo. 2017. La croisade " anti-genre ». Du Vatican au Manif pour Tous}

Julie Abbou

\section{OpenEdition}

Journals

Édition électronique

URL : http://journals.openedition.org/glad/1086

DOI : 10.4000/glad.1086

ISSN : 2551-0819

Éditeur

Association GSL

Référence électronique

Julie Abbou, «Sara Garbagnoli \& Massimo Prearo. 2017. La croisade " anti-genre ». Du Vatican au Manif pour Tous », GLAD! [En ligne], 04 | 2018, mis en ligne le 30 juin 2018, consulté le 17 décembre 2020.

URL : http://journals.openedition.org/glad/1086 ; DOI : https://doi.org/10.4000/glad.1086

Ce document a été généré automatiquement le 17 décembre 2020.

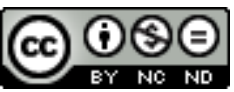

La revue GLAD! est mise à disposition selon les termes de la Licence Creative Commons Attribution -

Pas d'Utilisation Commerciale - Pas de Modification 4.0 International. 


\title{
Sara Garbagnoli \& Massimo Prearo. 2017. La croisade " anti-genre ». Du Vatican au Manif pour Tous
}

\author{
Julie Abbou
}

\section{RÉFÉRENCE}

Sara Garbagnoli \& Massimo Prearo. 2017. La croisade " anti-genre ». Du Vatican au Manif pour Tous. Paris : Éditions Textuel. 128 pages.

1 Le mouvement « anti-genre », visibilisé en France par La Manif pour Tous en 2013, a surpris par son ampleur soudaine. Passé le moment de stupéfaction qui a pu saisir le féminisme face à cette offensive réactionnaire, antiféministe, qui semblait surgir du passé, il importait de revenir sur la généalogie et les enjeux discursifs, idéologiques et sociaux de ce mouvement. C'est ce que fait cet ouvrage, en montrant qu'il ne s'agit en rien d'un mouvement spontané, d'un accident idéologique, mais au contraire d'une offensive aussi stratégique qu'idéologique, extrêmement préparée - et réussie - de la part du Vatican, qui lui a permis de se repositionner dans l'espace public tout en affirmant un nouveau discours interne. Ce repositionnement s'est principalement opéré par une refonte idéologique et argumentative sur la question du genre: «L'entreprise de contestation du concept de genre a pris la forme d'une nouvelle croisade catholique » (p. 12).

2 Véritable ouvrage de rhétorique, La croisade "anti-genre " offre un travail d'une rare précision sur la matérialité discursive du Vatican, en retraçant les lieux où se forment les discours, par qui, par quels déplacements discursifs, dans quel mouvement rhétorique, où et comment ils se diffusent. Le livre donne ainsi littéralement corps au discours " anti-genre » afin d'en faire la critique. Son objectif est en effet de dénoncer « l'avancée d'une vague réactionnaire qui, à partir de sa matrice catholique, se déploie sous la forme d'une contre-révolution sexuelle» (p. 16). 
3 Après une introduction générale qui permet d'asseoir le propos, le livre est composé en diptyque. La première partie, de Sara Garbagnoli, s'applique à détailler et à mettre au jour l'argumentaire " anti-genre », les axes de son dispositif discursif, et les processus de politisation de ce discours (p. 13). La seconde, de Massimo Prearo, cherche quant à elle, à comprendre comment les arguments de cette croisade se sont métamorphosés en mouvement social dans le passage à la rue. Ces deux aspects extrêmement complémentaires (fabrique discursive d'un renouveau idéologique et fabrique sociale d'une cause) offrent ainsi un tableau détaillé d'une offensive réactionnaire. Les deux parties partagent également une utilisation fine, informée et efficace des outils des SHS, non seulement pour produire une analyse théorique mais, en allant plus loin, pour contre-attaquer idéologiquement. Très informé, mais sans verbiage ni excès de citation pour se légitimer, le livre montre une réelle utilisation des concepts plutôt qu'un bardage et renoue ainsi avec les études féministes comme positionnement politique.

\section{Pourquoi et comment le Vatican s'en prend-il au genre ? (Sara Garbagnoli)}

Dans un texte clair, argumenté et incisif, Garbagnoli met à jour «l'invention puis la mise en circulation de l'objet "la théorie du genre" » comme une véritable politique discursive d'un ordre moral, qui repose sur la délégitimation des théories féministes et queers et sur le choix du concept de genre pour identifier un ennemi porteur d'une vision dénaturalisée de l'ordre sexué et sexuel (p. 10).

Le texte offre une solide démonstration que la binarité, l'essentialisation et la naturalisation ne sont pas des accidents idéologiques, mais bien des combats idéologiques. Garbagnoli en fait l'analyse en décortiquant les instances de diffusion doctrinaire, de production de textes, d'organisation de colloques, etc. qui vont servir de chambre de résonnance. Faire la critique de ce mouvement idéologique implique alors de se situer au carrefour du discursif et de l'idéologique, et d'assumer les enjeux rhétoriques de ce combat. C'est ce que fait l'auteure en menant un travail d'une grande précision factuelle sur les traces effectives de la matérialité discursive de l'idéologie anti-genre du Vatican, faisant apparaître sous nos yeux une histoire ultracontemporaine. La force du texte repose autant sur sa solidité intellectuelle que sa clarté politique, pour en faire une véritable réponse féministe à l'offensive réactionnaire du Vatican.

Après un rappel de la teneur du genre comme une révolution touchant aux catégories de perception du monde social les mieux naturalisés, (rappel d'ailleurs à la fois puissant et synthétique des apports du genre), Sara Garbagnoli montre que le Vatican «a bien saisi le potentiel révolutionnaire " que celui-ci recèle, comme "concept de combat » (p. 27) et qu'il « a obtenu ce dont les féministes rêvaient - faire du concept de genre un enjeu politique majeur»(p. 63-64). Il est donc nécessaire d'étudier les nouvelles positions argumentatives vaticanes, les énonciations contemporaines de son ordre moral, qui recourent à la promotion de la différence et de complémentarité entre sexe comme fondement de l'humain et à l'opposition au genre conçu comme instrument qui conduirait au règne du transhumanisme.

7 Garbagnoli contextualise et analyse ce renouveau argumentatif comme se produisant en deux mouvements : 1) l'euphémisation, par la création d'un pseudo-concept qui se 
superpose au genre ("idéologie du genre», "gender»), et 2) la déformation, notamment par des références fallacieuses à des théories, qui va permettre la diabolisation des théories adverses.

Les années 1940 et 1950 constituent "une discontinuité argumentative» dans le discours du Vatican qui abandonne l'ordre hiérarchique de la famille et la notion de naturelle soumission des femmes aux hommes pour lui préférer la notion de "complémentarité ", tout en maintenant la notion de "nature spécifique » des femmes, articulée à la question de la maternité. Ainsi, écrit l'auteure, la différence sexuelle porte un statut paradoxal de "marque indélébile à ne pas effacer ». Dans cette "opération antiféministe de police discursive ", la maternisation des femmes est le rouage principal d'une vision familialiste, paradigmatique sous les régimes vichystes et fascistes (p. 31). Dans les années 1960, ce glissement de la soumission à la complémentarité permet au Vatican de distinguer un bon féminisme d'un mauvais féminisme, plutôt que de s'y opposer frontalement dans un espace idéologique qui ne le permet plus. Il s'agit de dire que les injustices subies par les femmes consistent en l'empêchement de tenir leurs missions naturelles.

9 Sous le pontificat de Wojtyla (Jean-Paul II), les décennies suivantes (1980-2000) accentuent cette tendance par une surévaluation des vertus féminines : non seulement les femmes ont une dignité égale aux hommes, mais elles sont plus précieuses que ces derniers. Cela va s'accompagner d'une rupture doctrinale autour de la sexualité, qui n'est plus un péché, mais est réhabilitée comme une composante fondamentale de la personne, dans une théologie des corps. La conjugalité hétérosexuelle est ainsi pensée comme un don réciproque de soi entre deux espèces ontologiquement différentes, ce qui ouvrira la porte à la notion de « loi morale naturelle ", et permettra l'argument de "l'égalité dans la différence», dans une "tradition de racisation des femmes (...) comme une espèce, un "groupe naturel". (...) Cette torsion des notions féministes produit un discours qui, en se réclamant du féminisme, se déploie comme une arme antiféministe » (p. 38-39).

10 Enfin, sous Ratzinger (Benoît xVI), le mouvement d'euphémisation entrainera une distinction entre les bons homosexuels (chastes) et les mauvais (qui vivent leur homosexualité comme une identité politique) dans une doctrine de la bipolarité sexuelle. Le Vatican proposera alors la notion de " discrimination non injuste ».

11 À côté de cette entreprise d'euphémisation pour prendre place dans le débat et se rendre audible, l'appropriation et la torsion lexicale constituent également une contreattaque. Au début des années 2000 , le Vatican réagit à l'arrivée du concept de genre dans les politiques d'égalité (notamment de l'ONU), en créant une multitude d'instituts et d'académies, « des plateformes de scientifisation » de son discours théologique, avec «des actions de lobbying auprès des instances politiques internationales et des Parlements nationaux » (p. 45), et en produisant un ensemble d'outils rhétoriques de déformation qui vont lui permettre de structurer les cadres du débat, tout en s'affichant comme un discours séculier, scientifique et féministe qui repose sur la croyance dans la naturalité et dans la complémentarité entre les sexes (p. 58). Cette déformation discursive est menée à travers une prolifération des techniques de labellisation, qui fonctionnent comme des anathèmes et des signifiants déformants, que Garbagnoli détaille dans cette dernière section.

12 Elle décrit tout d'abord la création d'un répertoire de syntagmes («idéologie du genre ", "théorie du genre ", «idéologies des féministes du gender ", "théorie du 
genre sexuel ", "théorie du gender queer ", etc.), utilisé pour constituer un ennemi unique, et dont l'un des outils majeurs est le Lexique des termes ambigus et controversés sur la famille, la vie et les questions éthiques (2003). L'usage du mot théorie s'inscrit, lui, dans une stratégie anti-intellectualiste tout en proposant une opposition entre science et idéologie, pour défendre un déterminisme biologique et de la nature sexuelle des individus. Par ailleurs, l'usage du terme gender lui permet d'exotiser le concept au nom d'une critique de l'impérialisme culturel américain. Le Vatican recourt également à des mésusages conceptuels : le genre, renvoyant tantôt à la dimension sociale du sexe, tantôt au principe de hiérarchisation qui produit les sexes. Autre stratégie d'anathème, que Garbagnoli identifie comme la torsion la plus déformante: la diabolisation ad personam : «la théorie du genre affirmerait que genre et sexualité serait une affaire de "choix individuel" " dans une évaporation des hiérarchies. Ce brouillage du genre comme reflet du sexe est bien sûr au service d'une «tentative de renaturalisation du concept de genre » (p. 57). Elle affirme ainsi que la croisade « anti-genre » du Vatican prend la forme d'un double combat: «il ne s'agit pas seulement de délégitimer ce concept, mais aussi de le vider de sa charge critique » (p. 57-58).

Enfin, dernière déformation rhétorique étudiée: l'amphibologie, c'est-à-dire l'ambiguïté grammaticale que le Vatican entretient autour du terme genre qui désigne en fait simultanément : l'homosexualité, la famille, et la reproduction de l'ordre social (p. 59). Grâce à ce brouillage, il pourra présenter la famille hétérosexuelle comme la cellule de base de l'ordre national, en s'appuyant sur la mise en circulation des termes tels que « hétérophobie » ou «familiphobie » et construire un ennemi intérieur. Cette articulation du national, du familial et de la filiation lui permet de s'inscrire dans des rapports (de force?) aux États nationaux.

C'est là peut-être un des arguments les plus frappants du livre, et l'un des plus éclairants sur cette vague réactionnaire: avec l'intention «de vouloir garder (ou reprendre) le monopole du pouvoir d'établir la définition légitime de ce qu'est une famille - c'est-à-dire le groupe qui demeure le principal vecteur de transfert sexué et intergénérationnel du patrimoine - dans un contexte national donné »(p. 61), le Vatican se positionne en fait comme instance d'organisation et de gouvernance des vies, faute d'avoir gardé le gouvernement des sociétés, et s'affiche « non pas seulement comme une institution religieuse, mais aussi bien comme (...) l'autorité morale de référence de l'“Occident", (...) un modèle de nationalisme sexuel qui lie hétérosexualité, chrétienté, blanchitude et "identité nationale" » (p. 62).

\section{Un contre-mouvement sexuel (Massimo Prearo)}

15 La partie de Massimo Prearo s'ouvre par un questionnement : comment des milliers de personnes ont été amenées à descendre dans la rue pour défendre la famille hétérosexuelle ? C'est-à-dire comment la police discursive et argumentative décrite par Sara Garbagnoli dans la première partie a-t-elle pu se transformer en un processus efficace de mobilisation à échelle globale ? À partir d'observations des situations française et italienne, principalement dans les années 2010, Prearo met au jour la fabrique des possibilités d'un passage à l'acte du monde catholique, comme une politique sexuelle contre-révolutionnaire, mais aussi comme la réaffirmation identitaire d'un « nous catholique ». 

rhétorique à un dispositif de mobilisation, l'analyse procède en trois temps: (1) les foyers de production du savoir catholique, de ses acteurs et de ses outils, (2) les logiques de politisation de l'identité catholique par l'utilisation du discours "antigenre » comme propulseur d'une forme d'engagement militant, et (3) l'expression publique d'une sentiment de revanche identitaire, portée par la minorité des catholiques intégristes.

La première partie s'intéresse donc à la fabrique du corps théorique, cette fois-ci d'un point de vue sociologique : qui sont les producteurs de ce savoir, « au nom de qui et au nom de quoi construisent-ils/elles leur légitimité ? (...) avec quels outils, quels canaux? ? (p. 71). Prenant le relai de Garbagnoli, Prearo montre comment le Vatican s'est approprié «le vocabulaire de la démocratie sexuelle » (p. 72).

Prearo jette une lumière sociologique sur cette fabrique idéologique puisqu'il mène sa démonstration par un travail précis d'identification des acteurs (Marguerite Peeters, Dale O'Leary, Xavier Lacroix etc.), des institutions (think tanks, instituts, groupes de travail, etc.), des évènements, et des productions textuelles du Vatican des années 2000 qui vont servir à "réaffirmer les valeurs et les principes du modèle anthropologique catholique ». Ce travail sur les foyers discursifs lui permet de fonder son argument principal: le savoir "anti-genre » est une occasion «de restaurer la cohésion du catholicisme français autour d'affirmations doctrinales fortes » (p. 75), affirmations que Prearo analyse en deux moments : un moment identitaire, et un moment politique. Il prolonge et explicite ainsi la thèse amorcée dans la partie précédente : la volonté de l'Église de réaffirmer une autorité sur le gouvernement des conduites, vis-à-vis des États. transformer en activisme, puis en mobilisations de rue. En écho à Garbagnoli qui montrait qu'il s'agit d'une stratégie rhétorique, Prearo démontre, données précises à l'appui, que cette mobilisation n'est en rien une adhésion spontanée mue par une panique morale, mais correspond plutôt à une vaste campagne de recrutement et de formation par des experts : en 2013, des centaines de conférences sont organisées dans toute l'Italie, puis diffusées sur Internet, avec un cahier des charges méthodiquement préparé et un vocabulaire précis, parsemé de buzz words: d'abord un rappel du "fondement biologique, chromosomique, anthropologique de la différence sexuelle", puis «l'exposition des origines de "la théorie du genre" » et enfin la mention "des dangers réels » du gender qui « vise à instaurer un nouvel ordre mondial » pervers et un état totalitaire.

En France, le même mouvement se met en place dès 2011, avec la polémique des manuels scolaires de SVT. Là encore, des acteurs identifiés tels que Mgr d'Ornellas, Elizabeth Monfort, Christian Vanneste, etc. œuvrent au sein de groupes de travail et de think tanks néoconservateurs (l'Association pour la Fondation du Service politique, l'Académie d'éducation et d'études sociales, Famille \& Liberté, Alliance Vita, l'Opus Dei, etc.) et agissent au sein des paroisses mais aussi au-delà, un peu partout en France. Simultanément, une pratique réflexive catholique fournit un cadre de compréhension permettant la "remise en cause des fondements doctrinaires du catholicisme ", que Massimo Prearo désigne comme un aggiornamento réflexif de la morale catholique, et la fabrique d'une identité collective catholique renouvelée. C'est donc l'orchestration 
d'une panique morale que Prearo met au jour par l'identification et la contextualisation des acteurs collectifs et individuels.

Dans la troisième et dernière partie, Prearo propose de comprendre quels ont été les effets de cette mobilisation sur le militantisme catholique. Il avance en ce sens que «la cause "anti-genre" a reconfiguré le cadrage identitaire du militantisme religieux en contexte démocratique sécularisé » (p. 97). Son hypothèse, convaincante, est que l'incarnation du discours "anti-genre » dans les manifestations, a révélé et réactivé " une agency catholique, une capacité d'agir politiquement, à partir de son appartenance religieuse, mais au nom d'un combat "anthropologique" de défense de l'ordre naturel », ce qui a permis de penser l'identité catholique comme une identité politique (p. 101).

On retrouve là l'idée rencontrée plus tôt, que lorsque la religion veut gouverner alors qu'elle n'a plus d'emprise sur les États, elle est obligée de se faire plus ouvertement politique. Or, ce projet politique émane d'une tendance précise du catholicisme, à sa droite dure: le Chemin Néocatéchuménal. Prearo étudie les discours et positionnements de ces tendances pour dessiner les contours de leur projet idéologique comme profondément anti-démocratique, et contre-révolutionnaire, et la façon dont ils mettent en place un "nous", un moment de reconnaissance identitaire qui (se) manifeste. La présence ecclésiastique dans les mouvements « anti-genre » ne relève pas seulement de leur racine intégriste et orthodoxe, mais d'un travail identitaire sous une forme politisée qui permet d'affirmer « un catholicisme sans concessions, par ailleurs en perte d'hégémonie culturelle» (p. 105). C'est ce moment que l'auteur qualifie de revanche identitaire. Si la démonstration de cette affirmation identitaire et politique est très convaincante, la qualification de revanche identitaire, qui revient beaucoup, l'est un peu moins. On comprend bien que la revanche et l'offense dont parle Prearo sont des analogies pour le corps social catholique, mais le glissement métaphorique, qui psychologise, ne fonctionne que partiellement, surtout qu'a été montrée au préalable la complexité idéologique de l'institution catholique. Certes les discours « anti-genre » le formulent parfois en des termes proches (voir Tugdual Derville qui parle «du traumatisme provoqué par une dérive libertaire post-soixant-huitarde», p. 109), mais l'analyse critique gagnerait à replacer cela sur un terrain clairement politique (ce qui est fait par ailleurs dans les deux parties de l'ouvrage), sans glisser vers la métaphore psychologique. L'allégorie de "l'immeuble qui s'écroule» employée à l'occasion, fonctionne mieux pour comprendre comment l'Église catholique cherche à sauver les meubles idéologiques, dans un mouvement certes très offensif, mais pas nécessairement pensé - et vécu - comme la réponse à une perte morale à réparer.

Ce choix problématique du terme "revanche » est illustratif d'un registre accusateur, dans cette seconde partie, et dans une certaine mesure, d'un discours très imagé du dévoilement (Prearo parle de déguisement, d'infiltration, etc.), des effets de formule, qui certes sont frappants mais donnent parfois un sentiment de répétition, d'enchaînement de phrases chocs (par ailleurs très bien trouvées) et qui font par là perdre un peu de force au discours en l'inscrivant dans une stratégie dépréciative, qui fait parfois penser plus à l'essai qu'au texte politique. Mais c'est l'un des rares défauts du livre, et l'analyse compense largement l'effet terminologique.

L'ouvrage conclut sur l'idée forte que la construction du discours "anti-genre » du Vatican lui a permis de séculariser sa «morale sexuelle» (p. 113) et d'imposer dans l'agenda politique ses propres termes. La mobilisation a également permis aux mouvements ecclésiaux d'agir « en garants et défenseurs d'une identité catholique pure 
et dure » (p. 114), face à un catholicisme mou. Il s'agit donc d'un double mouvement, à l'adresse de l'espace public (séculier) mais aussi à l'intérieur de l'espace catholique. Cet argumentaire, tour de force rhétorique, révèle «le logiciel du système idéologique patriarcal et hétéronormatif avec ses hiérarchisations " par l'affirmation "audible et "démocratiquement" acceptable que les hommes et les femmes, les personnes hétérosexuelles et les personnes homosexuel-le-s et trans n'ont pas le droit à la même place dans le monde » (p. 115).

On pourra regretter des détails mineurs : à plusieurs reprises l'ouvrage rappelle qu'il se consacre uniquement aux discours du Vatican, qui ne sont pas les seuls dans l'arène des tenants de "la théorie du genre " et de cette refonte antiféministe. On regrette toutefois que ces autres discours ne soient pas mentionnés, au moins pour les situer. De même, quelques explications complémentaires sur les différentes mouvances catholiques mentionnées (mouvements ecclésiaux, néocatéchuménal, charismatiques, etc.) auraient aidé la lectrice profane, peu au fait des tendances internes au catholicisme, à être moins perdue dans cette cartographie. De manière plus formelle, le choix des titres de section n'est pas toujours limpide, ce qui produit une impression de redondance, d'accumulation, vite balayée par la clarté d'analyse des deux textes, leur solidité factuelle, leur perspicacité politique et leur grande complémentarité. Enfin, l'ouvrage a les défauts de ses qualités : si la focale discursive et la focale sociale mettent au jour des aspects différents du phénomène, ce découpage méthodologique produit parfois un effet de redondance, dans ces différentes analyses des discours de l'Église catholique. Mais cet effet est précisément ce qui permet d'éviter une division artificielle entre discursivité et société, ce qu'on ne peut que saluer.

Face aux débats sur la théorie du genre, deux types de réactions féministes se sont fait entendre : d'une part des voix s'élevaient pour assumer la portée théorique des études de genre et revendiquer le segment "théorie du genre", d'autre part des voix critiquaient la mise en circulation de cette notion. À travers l'analyse de la fabrique discursive, médiatique et évènementielle - et en fait performative - de «l'objet "la théorie du genre" ", cet ouvrage ${ }^{1}$ démontre brillamment que les termes mêmes du débat ont été conçus dans la visée d'une offensive anti-féministe à échelle mondiale, et qu'il est urgent de formuler une réponse féministe puissante. Si ce n'est chose faite, c'est au moins commencé.

\section{NOTES}

1. Un reflet de ces discussions s'est d'ailleurs glissé dans l'objet lui-même du livre, dont la couverture titre La croisade anti-genre, accompagné d'un carton d'erratum rappelant que : "le titre exact du livre est : La croisade "anti-genre" avec des guillemets ». 
INDEX

Thèmes : Actualités

Keywords : manif pour tous, Vatican, rhetoric, ideology, social movements

Mots-clés : manif pour tous, Vatican, rhétorique, idéologie, mouvements sociaux

\section{AUTEURS}

\section{JULIE ABBOU}

Titulaire d'un doctorat de Sciences du Langage (Aix-Marseille Université) sur les modifications du genre linguistique pour des motifs politiques, Julie Abbou mène des recherches sur les apports théoriques réciproques des études de genre et des sciences du langage. Elle travaille également sur les dimensions sémiotiques du genre grammatical, ainsi qu'en rhétorique sur le traitement du genre dans différents types de discours. Récemment, elle a co-dirigé l'ouvrage Gender, Language and the Periphery. Grammatical and social gender from the margins (John Benjamins) et publié des articles dans des revues telles que Semen, Mots les langages du politique, Current issues in Language Planning, etc. 